Preprints of the

Max Planck Institute for

Research on Collective Goods

Bonn 2009/19

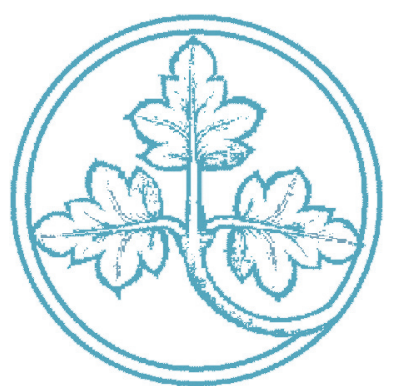

Rechtliche und ökonomische Rationalität im Emissionshandelsrecht

Stefan Magen

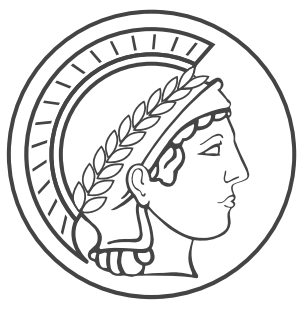




\title{
Rechtliche und ökonomische Rationalität im Emissionshandelsrecht
}

\author{
Stefan Magen
}

June 2009 


\section{Rechtliche und ökonomische Rationalität im Emissionshandelsrecht ${ }^{1}$}

Auf der Grundlage der Emissionshandels-Richtlinie ${ }^{2}$ wurde zum 1. Januar 2005 ein europaweites Handelssystem für Berechtigungen zur Emission von Kohlendioxid $\left(\mathrm{CO}_{2}\right)^{3}$ eingeführt. ${ }^{4}$ Dieses gilt als marktwirtschaftliches Steuerungsinstrument. ${ }^{5}$ Es ist nach der Blaupause eines in den Wirtschaftswissenschaften entworfenen Steuerungsmodells konstruiert, nämlich nach dem sog. cap-and-trade Ansatz der Umweltökonomie. ${ }^{6}$ Als Steuerungsmechanismus fungiert dabei ein Markt für handelbare Emissionsrechte, der dafür sorgt, dass Emissionen zu den geringsten Kosten vermieden werden. Derartige ökonomische Regulierungsinstrumente werfen die Frage auf, was ihre Umsetzung für die Eigenrationalität des Rechts bedeutet. ${ }^{7}$ Betrachtet man das Treibhausgasemissionshandelsgesetz (TEHG) und die beiden Zuteilungsgesetze (ZuG 2007 und ZuG 2012) unter diesem Blickwinkel, könnte allerdings der Eindruck entstehen, im deutschen Emis-

1 Für sehr hilfreiche Anregungen und Kritik danke ich Christoph Engel, Martin Hellwig, Gregor Kirchhof, Karsten Schneider und Emanuel Towfigh.

2 Richtlinie 2003/87/EG des Europäischen Parlaments und des Rates über ein System für den Handel mit Treibhausgasemissionszertifikaten in der Gemeinschaft und zur Änderung der Richtlinie 96/61/EG des Rates v. 13. Oktober 2003, ABl. L 275 vom 25. Oktober 2003, zuletzt geändert durch RL 2004/101/EG des Europäischen Parlaments und des Rates vom 27. Oktober 2004, ABl. 2004 L 338.

3 Die Emissionshandelsrichtlinie gilt zwar nach Anhang II auch für die Emission anderer Treibhausgase, erfasst aber gemäß Anhang I als Tätigkeit bislang nur die Emission von Kohlendioxid.

4 In der ersten Handelsperiode 2005-2007 waren an diesem europäischen Emissionshandelssystem ca. 11.400 Anlagen beteiligt, die jährlich ca. 2,2 Milliarden Tonnen Kohlendioxid emittierten, was ca. $45 \%$ der europäischen Gesamtemissionen entsprach ( $R$. Kühne, Immissionsschutz durch Emissionshandel - eine Zwischenbilanz, LKV 16 [2006], 356 [358]). In Deutschland stand man handelbaren Emissionszertifikaten zuvor bekanntermaßen skeptisch gegenüber; vgl. M. Kloepfer, Der Handel mit Emissionsrechten im System des Umweltrechts, in: R. Hendler et al. (Hg.), Emissionszertifikate im Umweltrecht (UTR Bd. 74), 2004, 71 (84); R. Sparwasser/R. Engel/A. Voßkuhle, Umweltrecht, 5. Aufl. 2003, § 2 Rn. 127.

5 Sachverständigenrat für Umweltfragen, Die nationale Umsetzung des europäischen Emissionshandels: Marktwirtschaftlicher Klimaschutz oder Fortsetzung der energiepolitischen Subventionspolitik mit anderen Mitteln, 2006; H. Bonus/H. Niebaum, Voraussetzungen für Umweltzertifikate in Deutschland, in: H. Bonus (Hg.), Umweltzertifikate, 1998, 225 (226); C. Knüll, Grundrechtliche Probleme der Allokation von CO2Zertifikaten, 2009, 42 ff.; kritisch C. Weidemann, Emissionserlaubnis zwischen Markt und Plan, DVBl. 2004, 727 (es handele sich um ein ,ökonomisch wirkendes Zwangssystem des Umweltstaates, nicht um ein marktwirtschaftliches Instrument").

6 Grundlegend Th. D. Crocker, The Structuring of Atmospheric Pollution Control Systems, in: H. Wolozin (Hg.), The Economics of Air Pollution, 1966, 61 ff.; J. H. Dales, Land, Water, and Ownership, Canadian Journal of Economics 1 (1968), 791 (801 ff.); ders., Pollution, Property and Prices: An Essay in PolicyMaking and Economics, 1968; W. D. Montgomery, Markets in Licences and Efficient Pollution Control Programs, Journal of Economic Theory 5 (1972), 395 ff.; T. H. Tietenberg, Emissions Trading: An Exercise in Reforming Pollution Policy, 1985; für Überblicke vgl. D. Cansier, Umweltökonomie, 2. Aufl., 1996, 187 ff.; A. Endres, Umweltökonomie, 3. Aufl. 2007, 110 ff.; Knüll (Fn. 5), 39 ff.; K. L. Mehrbrey, Verfassungsrechtliche Grenzen eines Marktes handelbarer Emissionsrechte, 2003, 21 ff.; Th. Sterner, Policy Instruments for Environmental and Natural Resource Management, 2003, 82 ff.; J. Weimann, Umweltökonomik, 3. Aufl. 1995, 226 ff.; wichtige ökonomische Aufsätze sind wieder abgedruckt bei T. H. Tietenberg, Emissions Trading Programs. Bd. 1: Implementation and Evolution, Bd. 2: Theory and Design, 2001.

7 Zum Umweltrecht U. Di Fabio, Ökonomisierung des Umweltrechts, in: R. Hendler et al. (Hg.), Jahrbuch des Umwelt- und Technikrechts 2006 (UTR Bd. 90), S. 251 (257); für eine parallele Diskussion im Kartellrecht vgl. W. Möschel, Juristisches versus ökonomisches Verständnis eines Rechts der Wettbewerbsbeschränkungen, in: Festschrift Tilmann, 2003, 705 (715 ff.). Grundsätzlich zur Ökonomisierung des Verwaltungsrechts J.-P. Schneider, Zur Ökonomisierung von Verwaltungsrecht und Verwaltungsrechtswissenschaft, Die Verwaltung 34 (2001), 317 ff.; zur Ökonomisierung der Verwaltungsorganisation A. Musil, Wettbewerb in der staatlichen Verwaltung, 2005; G.-F. Schuppert, Verwaltungsorganisation und Verwaltungsorganisationsrechts als Steuerungsfaktoren, in: W. Hoffmann-Riem/E. Schmidt-Aßmann/A. Voßkuhle (Hg.), GVwR, Bd. I, 2006, § 16, Rn. 110 ff.; zu ökonomischen Anreizen U. Sacksofsky, Anreize, in: W. Hoffmann-Riem/E. Schmidt-Aßmann/A. Voßkuhle (Hg.), GVwR, Bd. II, 2008, § 40. 
sionshandelsrecht sei eher die ökonomische Rationalität von der rechtlichen verdrängt worden. Spezifisch ökonomische oder marktwirtschaftliche Regelungen sind dort auf den ersten Blick jedenfalls kaum auszumachen. Stattdessen stößt man auf das bekannte Regulierungsinstrumentarium der hoheitlichen Eingriffsverwaltung: Ordnungsrecht, Planungsrecht und Bewirtschaftungsregeln. ${ }^{8}$ Als Folge dieser ,eigentümlichen Mischung von genuin öffentlich-rechtlichem Instrumentarium und ökonomischer Steuerung ${ }^{* 9}$ werden auch die Rationalitäten von Ökonomie und Recht vermengt. Es fragt sich deshalb, wie genau beide Rationalitäten im Emissionshandelsrecht zusammenwirken, konfligieren oder nebeneinander bestehen.

\section{Rechtliche und ökonomische Rationalität}

Die Unterscheidung von rechtlicher und ökonomischer Rationalität gewinnt ihre Bedeutung aus dem Umstand, dass in modernen Gesellschaften Recht und Wirtschaft relativ eigenständige soziale Ordnungen sind, die mit je eigenen Institutionen unterschiedliche gesellschaftliche Funktionen erfüllen. ${ }^{10}$ Man kann sich lange darüber streiten, was genau die Funktion der jeweiligen Ordnung ist, ob es sich um eine oder mehrere handelt und ob und in welchem Sinn man diese Ordnungen als „Systeme“ beschreiben kann. ${ }^{11}$ Dass es sich aber im Grundsatz um ausdifferenzierte Ordnungen handelt, die unterschiedlichen Funktionen dienen, ist im Kontrast betrachtet unübersehbar. Für die hiesigen Zwecke kann man ihre jeweiligen Funktionen so beschreiben, dass Wirtschaft die Funktion hat, mit Hilfe von Märkten die Produktion und Verteilung knapper Güter zu organisieren. Die Funktion des Rechts kann man darin sehen, durch Setzung bzw. Anerkennung von Normen sowie deren autoritative Anwendung und Durchsetzung eine relativ stabile Koordination der wechselseitigen normativen Erwartungen zu schaffen. Dazu gehört dann auch, anderen sozialen Ordnungen einen rechtlichen Rahmen bereitzustellen und Konflikte autoritativ zu entscheiden. Die Verschiedenheit der Funktionen impliziert nun auch, dass die Akteure ihr Handeln im Kontext der jeweiligen Ordnung - nicht ausschließlich, aber doch prägend - an unterschiedlichen Leitwerten ausrichten. Im ökonomischen Kontext sind dies insbesondere Eigeninteresse und Effizienz, im Recht dagegen ein Bündel von Rechtswerten, zu denen insbesondere Freiheit, Rechtsförmlichkeit, Gemeinwohl und ein gerechter Interessenaustausch gehören. ${ }^{12}$ Mit ökonomischer Rationalität ist hier dann die Eigenschaft von Märkten und anderen ökonomi-

Vgl. R. Breuer, Umweltschutzrecht, in: E. Schmidt-Aßmann/F. Schoch (Hg.), Besonderes Verwaltungsrecht, 14. Aufl. 2008, S. 591 ff., Rn. 222h; Ch. Enders, Die Inkorporation des Emissionshandels in das deutsche Luftreinhaltungsrecht - normativer Rahmen und aktuelle Rechtsprobleme, LKV 17 (2007), 193 (194); Di Fabio (Fn. 7), 255; M. Kloepfer, Umweltschutz zwischen Ordnungsrecht und Anreizpolitik, Zeitschrift für angewandte Umweltforschung 9 (1996), 56 (64 u. 203); M. Schmidt-Preuß, Flexible Instrumente des Umweltschutzes, in: Gesellschaft für Umweltrecht (Hg.), Umweltrecht im Wandel, 2001, 319; I. Spiecker gen. Döhmann, Das Verwaltungsrecht zwischen klassischem dogmatischem Verständnis und steuerungswissenschaftlichem Anspruch, DVB1. 2007, 1074 (1079).

$9 \quad$ Enders (Fn. 8), 194.

10 Ausführlich zur Rolle von Institutionen Ch. Engel, Institutionen zwischen Staat und Markt, Die Verwaltung 34 (2001), 1 (5 ff.).

11 U. Schimank, Theorien gesellschaftlicher Differenzierung, 1996; ders./U. Volkmann, Gesellschaftliche Differenzierung, 1999. Für eine pointierte Sicht vgl. N. Luhmann, Das Recht der Gesellschaft, 1995; ders., Die Wirtschaft der Gesellschaft, 1988. 
schen Institutionen gemeint, durch Anreize eine effiziente Produktion und Verteilung knapper Güter zu erreichen. Rechtliche Rationalität bezeichnet hier dagegen die Eigenschaft des Rechts, Rechtswerte zu verwirklichen und eine relativ stabile Koordination normativer Erwartungen zu erreichen.

Der gesellschaftlichen Ausdifferenzierung korrespondiert nun auch eine fachliche Spezialisierung der Berufsträger. Insoweit verfolgen Juristen und Ökonomen - nicht ausschließlich, aber doch prägend - unterschiedliche Ziele und Werte und sie verfügen über eigene Kompetenzen, die auf die Realisierung ihrer jeweiligen Ziele und Werte ausgerichtet sind. Für die jeweiligen Disziplinen stellt sich deshalb die Frage, wie sie mit der Vermengung beider Rationalitäten im Emissionshandel umgehen sollte. Müssen sich etwa Rechtsprechung und Rechtswissenschaften um ein Verständnis der ökonomischen Rationalität bemühen oder diese gar berücksichtigen? Und welche spezifischen Kompetenzen bringen Ökonomie und Jurisprudenz insoweit ein? Die Antwort auf letztere Frage ist insbesondere für die Jurisprudenz nicht offensichtlich. Das gilt umso mehr, wenn man sich fragt, welchen Beitrag die Jurisprudenz denn dazu leistet, dass das, was später als Eigenrationalität des Rechts sichtbar wird, in den Prozess der Rechtssetzung Eingang findet. Spezialisiert hat sich die Jurisprudenz, so scheint es, auf die Auslegung und Anwendung von Gesetzen mit den Methoden juristischer Interpretation. Das hilft aber im Prozess der Rechtssetzung nicht viel weiter. Sicherlich eine juristische Kompetenz ist die Frage der Verfassungsmäßigkeit einer Regelung, aber auch sie bleibt auf eine Kontrollperspektive beschränkt. Bleibt dann nur noch die Aufgabe, das von der Ökonomie formulierte Steuerungsmodell formgerecht zu protokollieren? Oder besitzt die Jurisprudenz eigene Kompetenzen in der Umsetzung von Rechtswerten, in Fragen der Rechtsstaatlichkeit, der Freiheitlichkeit, der rechtlichen Verfolgung von Gemeinwohlzielen und des gerechten Interessenausgleichs?

Diese Fragen sind zu umfassend, als dass sie im Folgenden erschöpfend behandelt werden könnten. Die Erörterungen konzentrieren sich deshalb auf zwei Aspekte, die für das Emissionshandelsrecht charakteristisch sind. Der erste Aspekt betrifft das eigentümliche Nebeneinander von Marktfreiheit und ordnungsrechtlichem Eingriffsinstrumentarium (sogleich II.), der zweite die Zuteilungsregeln und die Notwendigkeit, bei deren rechtlicher Ausgestaltung und Interpretation die ökonomischen Wirkungen und Funktionen zu berücksichtigen (unten III.). Ein Hinweis erscheint insoweit an dieser Stelle aber schon vorab sinnvoll. Ökonomie und Jurisprudenz denken und beschreiben in unterschiedlichen, inkongruenten Kategorien. Diese Inkongruenz der Ordnungen oder Disziplinen verdeckt aber leicht, dass in der Sache beide gar nicht so selten nicht oder nur begrenzt im Konflikt liegen. Ein gutes Beispiel dafür ist das Konzept der Effizienz. Eine anspruchsvolle rechtsphilosophische und rechtswissenschaftliche Literatur ist mit dem Nachweis beschäftigt, dass Effizienz als normative Forderung auf schwachen Füßen steht und dass die vielfältigen Zwecke des positiven Rechts nicht auf Effizienz reduziert werden können. ${ }^{13}$ Was dabei leicht übersehen wird, ist, dass man vieles von dem, was die Ökonomie als Effizienzproblem konzeptualisiert, verfassungsrechtlich in die Kategorie des legitimen Gemeinwohlbelangs 
übersetzen kann. Michael Anderheiden hat das vor noch nicht so langer Zeit im Rahmen einer Assistententagung dargelegt, und zwar mit Hilfe des ökonomischen Konzepts der kollektiven Güter. $^{14}$

\section{Das Emissionshandelsrecht als Umweltordnungsrecht mit Marktenklave}

\section{Marktfreiheit und hoheitlicher Zwang}

Mit dem (partiellen) Wechsel vom Ordnungsrecht zum Emissionshandel war mitunter die Erwartung eines Zugewinns an marktwirtschaftlicher Freiheit verbunden. ${ }^{15}$ Die Realität des mittlerweile errichteten Emissionshandelssystems muss für solche Hoffnungen eine gewisse Ernüchterung sein. Der Emissionshandel hat nämlich das ordnungsrechtliche Eingriffsinstrumentarium keineswegs abgeschafft. ${ }^{16}$ Auch zur Emission von $\mathrm{CO}_{2}$ bedarf der Anlagenbetreiber einer Genehmigung, nämlich der Emissionsgenehmigung (§ 4 TEHG). Diese stellt ein klassisches präventives Verbot mit Erlaubnisvorbehalt dar und dient der Kontrolle öffentlich-rechtlicher Betreiberpflichten (§§ 5, 6 TEHG), ${ }^{17}$ deren Einhaltung von Verwaltungsbehörden überwacht und ggf. mit Zwangsmaßnahmen und Sanktionen durchgesetzt wird (§§ 17-21 TEHG). Dem Inhalt nach unterscheiden sich die Betreiberpflichten des TEHG allerdings von denen nach dem BImSchG. ${ }^{18}$ Statt in Gestalt von Grenzwerten materielle Vorsorgeanforderungen vorzugeben, ${ }^{19}$ konstatiert das TEHG nur prozedurale Betreiberpflichten. Deren Funktion ist es, sicherzustellen, dass jeder Betreiber nicht mehr emittiert, als er durch Emissionsberechtigungen abdecken kann. Deshalb verpflichtet das Emissionshandelsrecht die Betreiber, ihre jährlichen $\mathrm{CO}_{2}$-Emissionen nach vorgegebenen Methoden ${ }^{20}$ selbst zu ermitteln und nach Verifikation durch einen anerkannten Sachverständigen der zuständigen Behörde zu berichten ( $\$ 5$ TEHG). Eine dogmatische Neuerung ist die emissionshandelsrechtliche „Kardinalpflicht“ des $\S 6$ I TEHG, der die Betreiber dazu verpflichtet, beim Umweltbundesamt (Deutsche Emissionshandelsstelle) Emissionsberechtigungen

14 M. Anderheiden, Ökonomik, Gemeinwohl und Verfassungsrecht, in: M. Bungenberg et al. (Hg.), Recht und Ökonomik, 113 (120 ff.); ders., Gemeinwohlförderung durch Bereitstellung kollektiver Güter, in: W. Brugger et al. (Hg.), Gemeinwohl in Deutschland, Europa und der Welt, 2002, 391 ff.; ders., Gemeinwohl in Republik und Union, 2006, $110 \mathrm{ff}$.

15 Z. B. Bonus/Niebaum (Fn. 5), 226. Kritisch zur Gleichsetzung von Markt und Freiheit bzw. Staat und Zwang Engel (Fn.10), 5.

16 Konzise Überblicke z. B. bei K. F. Gärditz, Einführung in das Klimaschutzrecht, JuS 2008, 324 (326 f.); R. Schmidt/W. Kahl, Umweltrecht, 7. Aufl. 2006, § 3 Rn. 152 ff.

17 R. Körner, in: R. Körner/H.-P. Vierhaus (Hg.), TEHG, 2005, § 4 Rn. 19; kritisch aber Enders (Fn. 8 ), 195 f.

18 Nach § 5 I 2-4 BImSchG konkretisiert das TEHG insoweit die Pflicht des Anlagenbetreibers zur Vorsorge und zur Energieverwendung (H. D. Jarass, BImSchG, 7. Aufl., 2007, § 5 Rn. 68a und 104a). Die Schutzpflicht des $\S 5$ I 1 Nr. 1 BImSchG bleibt hingegen bestehen und kann zumindest theoretisch nach $\S 5$ I 3 BImSchG auch durch behördliche Anordnungen durchgesetzt werden. Ausführlich zum Verhältnis der Betreiberpflichten nach dem TEHG und dem BImSchG T. Hohmuth, Emissionshandel und deutsches Anlagenrecht, 2006, S. 260 ff.; zum Inhalt der Vorsorgepflicht D. Couzinet, Die Zulässigkeit von Immissionen im anlagenbezogenen Immissionsschutzrecht, 2007, $397 \mathrm{ff}$.

19 Zu Leistungsfähigkeit von Grenzwerten aus steuerungstheoretischer Sicht vgl. Ch. Engel, Die Grammatik des Rechts, in: H.-W. Rengeling/H. Hof (Hg.), Instrumente des Umweltschutzes im Wirkungsverbund, 17 (34 ff.).

20 H.-P. Vierhaus/S. v. Schweinitz, in: Körner/Vierhaus (Fn. 17), TEHG, § 5 Rn. 29 ff. 
in Höhe der im Vorjahr freigesetzten Emissionen abzugeben. Diese Berechtigungen kann der Betreiber entweder auf dem Emissionsmarkt erworben haben oder sie wurden ihm vor Beginn der einzelnen Handelsperioden (2005-2007 und 2008-2012) auf der Grundlage eines Zuteilungsgesetzes durch Verwaltungsakt ${ }^{21}$ kostenlos zugeteilt ( 9 TEHG). Sowohl das Gesamtkontingent

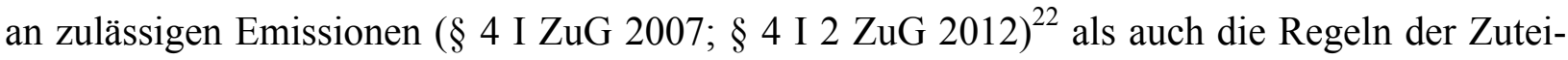
lung ( $\S 7$ ff. ZuG 2007, $\S 6$ ff. ZuG 2012) werden dabei vom Staat einseitig-hoheitlich festgelegt, und zwar durch die Zuteilungsgesetze auf der Grundlage eines Nationalen Allokationsplans (§ 7 TEHG). Zutreffend konstatiert deshalb Udo Di Fabio: „Das deutsche Emissionshandelssystem mit den Stellschrauben der Verknappung, aber auch der spezifischen Zuteilungen nach einem politisch entschiedenen Zuteilungskonzept, die Organisationsstruktur der Deutschen Emissionshandelsstelle und der dem Anlagenbetreiber zugemutete Prozesszyklus erinnern jedenfalls bei erster Betrachtung weit mehr an Planwirtschaft als an einen frei atmenden Markt.“23

Dieser Eindruck hält nicht nur einem vertieften zweiten Blick stand. Es zeigt sich sogar, dass der Charakter des Emissionshandelsrechts als ein primär hoheitliches Eingriffsrecht in der ökonomischen Logik des Emissionshandelsmodells selbst angelegt ist. Die Erwartung marktwirtschaftlicher Freiheit ist dabei nicht grundsätzlich falsch, sie ist aber nur für den engen Bereich des Handels mit Emissionsrechten berechtigt. Die diesen Freiheitszuwachs ermöglichende Übertragbarkeit der individuellen Emissionsbefugnis wird aber paradoxerweise erst durch eine Intensivierung hoheitlich-administrativer Regulierung der Emissionstätigkeit möglich. ${ }^{24}$ Den Grund dafür beschreibt die ökonomische Theorie selbst, nämlich mit dem Konzept der kollektiven Güter. Kollektive Güter sind ökonomisch betrachtet Fälle von Marktversagen, das heißt Konstellationen, in denen auch nach der ökonomischen Logik eine direkte oder indirekte staatliche Intervention gefordert ist. ${ }^{25}$ Das gilt auch für den Klimaschutz. ${ }^{26}$ Weil die Atmosphäre zunächst einmal jeder emittierenden Anlage zur Entsorgung ihrer Treibhausgasemissionen offen steht, braucht es Zwang oder Macht, damit die Anlagenbetreiber auf die Belange der Allgemeinheit Rücksicht nehmen und ihre Treibhausgasemissionen reduzieren. ${ }^{27}$ Der Emissionshandel befreit also das Recht nicht von der Aufgabe, Treibhausgasemissionen wegen ihrer negativen Externalitäten durch hoheitlichen Zwang zu begrenzen. Aus diesem strukturellen Grund bleibt das Emissionshandelsrecht im Kern Umweltordnungsrecht. Diese Zielsetzung unterscheidet das Emissionshandelsrecht auch von anderen Formen der staatlichen Regulierung von Märkten, insbesondere von dem Wettbewerbsrecht und dem Recht der Netzregulierung. Letztere wollen primär die Funkti-

$21 \quad$ Breuer (Fn. 8), Rn. 222f.

22 Es betrug 859 Mio. t CO2 pro Jahr in der Handelsperiode 2005-2007 und 844 Mio. t CO2 pro Jahr in der Handelsperiode 2008-2012.

23 Di Fabio (Fn. 7), 255; für weitere Stellungnahmen in diese Richtung siehe oben, Fn. 8.

24 Vgl. Breuer (Fn. 8), Rn. 222h.

25 Ch. B. Blankart, Öffentliche Finanzen in der Demokratie, 7. Aufl. 2008, 51 ff.; M. Fritsch/Th. Wein/H.-J. Ewers, Marktversagen und Wirtschaftspolitik, 7. Aufl. 2007, 364 ff. Dabei darf allerdings nicht übersehen werden, dass auch der Markt auf staatlich gesicherte Institutionen angewiesen sein kann, nämlich auf Verfügungsrechte; vgl. Engel (Fn.10), 5 ff.; R. Richter/E. G. Furubotn, Neue Institutionenökonomik, 3. Aufl., 2003, $86 \mathrm{ff}$.

26 R. Menges, Verteilungs- und Allokationswirkungen energiepolitischer Instrumente, ZNER 11 (2007), 285; T. Tietenberg, Environmental Econmics and Policy, 4. Aufl., 2004, 299.

Weimann (Fn. 6), 226. 
onsfähigkeit privater Gütermärkte erhalten oder herstellen. ${ }^{28}$ Das Emissionshandelsrecht dagegen hat zunächst einmal die Aufgabe, durch hoheitliche Oktroyierung eines begrenzten Emissionskontingents die Menge der Treibhausgasemissionen zu senken. Der Emissionsmarkt dient dabei nur als Hilfsmittel, um Kosten einzusparen und technische Innovationen anzuregen. ${ }^{29}$ Bereits der kanadische Ökonom John H. Dales, der gemeinhin als Erfinder des Modells handelbarer Verschmutzungsrechte angesehen wird, hat auf diese Eigenheit des Emissionsmarktes hingewiesen: ${ }^{30}$ „The market in pollution rights is not a ,true' or ,natural' market. [It] is nothing more than an administrative tool“" 31

Dass das Emissionshandelsrecht zunächst das Emissionsniveau durch Zwangsmaßnahmen begrenzt, widerspricht also der ökonomischen Rationalität nicht, sondern ist im Gegenteil eine Forderung der Effizienz im Sinne der sozialen Wohlfahrt. ${ }^{32}$ Ebenso wie das Umweltordnungsrecht enthält der Emissionshandel aber keinen Mechanismus, der sicherstellen würde, dass die gesellschaftlichen Kosten für die Eingrenzung der Treibhausgasemissionen geringer sind als der Nutzen der Vermeidung von Klimaschäden, sich also insgesamt ein effizientes Niveau an Emissionen einstellt. ${ }^{33}$ Das hängt nämlich entscheidend von der Festlegung des Gesamtkontingents an Berechtigungen ab, die aber, wie gesagt, als eine politisch-planerische Entscheidung vom Staat getroffen wird. Seinen spezifischen Beitrag zur Erhöhung der Effizienz leistet der Emissionsmarkt an einer anderen Stelle, nämlich darin, ein gegebenes Emissionsniveau mit möglichst geringen Vermeidungskosten zu erreichen. ${ }^{34}$ Die regulatorischen Maßnahmen, die erforderlich sind, um die dafür notwendigen Rahmenbedingungen zu schaffen, erhöhen allerdings die Intensität der staatlichen Freiheitseingriffe. Um aus der Emissionsbefugnis ein handel- und quantifizierbares privates Gut zu machen, müssen nämlich die individuellen Emissionen so detailliert überwacht werden, dass die Behörde exakt weiß, in welchem Umfang der einzelne Betreiber dieses Gut konsumiert hat. Das stellen die bereits erwähnten Ermittlungs- und Berichtspflichten sicher, die $\S 5$ TEHG den Betreibern auferlegt. Zu dem direkten hoheitlichen Zwang, den auch das Immissionsschutzrecht braucht, kommen also die mittelbaren Zwänge der regulierten Selbstregulierung hinzu, die nicht übersehen werden sollten. ${ }^{35}$ Die Kosten dafür und für die Teilnahme

28 Fritsch/Wein/Ewers (Fn. 25), 225 ff.; F. Höffler, Regulierung von Netzindustrien aus ökonomischer Sicht, in: J. Lüdemann (Hg.), Telekommunikation, Energie, Eisenbahn, 2008, 3 ff.

29 Das Emissionshandelssystem werde geschaffen, um ,auf kosteneffiziente und wirtschaftlich effiziente Weise auf eine Verringerung von Treibhausgasemissionen hinzuwirken“ (Art. 1 RL 2003/87/EG) bzw. um „durch eine kosteneffiziente Verringerung von Treibhausgasen zum weltweiten Klimaschutz beizutragen.“ (§ 1 I 1 TEHG).

30 Neben Thomas D. Crocker; vgl. Sterner (Fn. 6), 82; Crocker (Fn. 6).

31 Dales (Fn. 6), 803 f.; vgl. auch M. L. Weitzman, Prices vs. Quantities, Review of Economic Studies 41 (1974), 477 (490).

32 Erst das staatliche Verbot freier Emissionen schafft zudem die Knappheit, ohne dies es keine Nachfrage nach Emissionsrechten gäbe; Weimann (Fn. 6), 226; R. Schmidt/W. Kahl, Umweltrecht, 7. Aufl. 2006, § 3 Rn. 150.

33 Weimann (Fn. 6), 228. Der einflussreiche Klimabericht des britischen Ökonomen Nicholas Stern (sog. Stern Review) kommt diesbezüglich zu dem Ergebnis, dass die gegenwärtigen Emissionsreduktionen insoweit zumindest deutliche Effizienzverbesserungen darstellen; vgl. N. Stern, The Economics of Climate Change, 2007, S. $649 \mathrm{ff}$.

$34 \quad$ Endres (Fn. 6), $131 \mathrm{f}$.

35 Vgl. Gärditz (Fn. 16), 327; aus der Sicht der Praxis I. Zenke/Th. Fuhr/M. Bornkamm (Hg.), CO2-Handell aktuell, 2009, 95 ff.; aus Sicht der betroffenen Unternehmen B. Ortlieb, Der Emissionshandel in Deutschland - Erfahrungen und Erwartungen, in: M. Oldiges (Hg.), Immissionsschutz durch Emissionshandel - eine Zwi- 
am Emissionshandel sollen sich je nach Größe des Unternehmens zwischen einigen Hunderttausend und über einer Million Euro bewegen. ${ }^{36}$ Zum Teil sind diese Belastungen vielleicht die Folge einer übermäßig bürokratischen Umsetzung des ökonomischen Steuerungsmodells. Der Tendenz nach stellen sie aber keinen Widerspruch zur ökonomischen Rationalität dar, sondern deren Konsequenz. Als Übergang vom Ordnungsrecht zur Marktwirtschaft kann man den Emissionshandel deshalb nicht beschreiben. Er gleicht eher einer bewirtschafteten Marktenklave, die als Anreiz für Kosteneinsparung und Innovation innerhalb des Umweltordnungsrechts eingerichtet wird. $^{37}$

\section{Der Beitrag des Emissionshandels zum Klimaschutz}

Aus dem Gesagten folgt nun auch, dass der Emissionshandel als solcher keinen direkten Beitrag zum Klimaschutz erbringt. Denn unter einem Emissionshandelssystem richtet sich die Höhe der Emissionen nach dem zu Beginn einer Handelsperiode planerisch festgesetzten Kontingent an Berechtigungen, dessen Einhaltung vermittels der öffentlich-rechtlichen Betreiberpflichten hoheitlich durchgesetzt wird. ${ }^{38}$ Solange nicht Berechtigungen aus dem Markt genommen werden oder ungenutzt bleiben, haben weder die Zuteilungsmethode noch die durch sie bewirkte anfängliche Verteilung, irgendeinen Einfluss auf das Klimaschutzziel. Der Emissionsmarkt sorgt vielmehr dafür, dass die an einer Stelle eingesparten Emissionsberechtigungen an anderer Stelle wieder eingesetzt werden. Deshalb sind besondere Klimaschutzanstrengungen einzelner Betreiber oder Betreibergruppen für den Klimaschutz unerheblich. ${ }^{39}$ Sie verändern nur die Verteilung der Klimaschutzanstrengungen zwischen den emissionshandelspflichtigen Anlagen. Der Emissionsmarkt kann dadurch sogar Klimaschutzanstrengungen konterkarieren, wenn emissionshandelspflichtige Produktionsweisen durch Tätigkeiten ersetzt werden, die mangels Emissionen nicht dem Emissionshandel unterliegen. Insbesondere bei der Förderung erneuerbarer Energien kann dies zum Problem werden. ${ }^{40}$ Ersetzt etwa ein Stromversorger Kohlestrom durch Windenergie, spart er dadurch zugleich Emissionsberechtigungen ein. Der Emissionsmarkt sorgt dann aber dafür, dass die durch Windenergie eingesparten Emissionsberechtigungen an anderer Stelle wieder eingesetzt werden können, zum Beispiel zur Kohleverstromung im Nachbarland. Man stelle sich vor, die Feuerwehr wollte einen vollgelaufenen Keller mit einem Schlauch leerpumpen, dessen Ende durch das nächste Fenster in den gleichen Keller wieder hineinführt. Das schließt nicht aus, dass es andere Gründe geben mag, erneuerbare Energien zu subventionieren, zum Beispiel

schenbilanz, 2007, 251 ff.; zu den sog. Transaktionskosten des Emissionshandels siehe auch Knüll (Fn. 5), $59 \mathrm{f}$.

36 So die Schätzung von W. Ströbele, Klimapolitik: Kyoto-Protokoll und Emissionshandel für CO2-Zertifikate in der EU, Perspektiven der Wirtschaftspolitik 3 (2005), 325 (341). Die Grundlage dieser Schätzungen wird dort allerdings nicht genannt.

37 Vgl. Enders (Fn. 8), 194: „Bewirtschaftung nach Marktgrundsätzen“.

38 M. Burgi/P. Selmer, Verfassungswidrigkeit einer entgeltlichen Zuteilung von Emissionszertifikaten, 2007, 65; Cansier (Fn. 6), 194 f.; Knüll (Fn. 5), 48 f.

39 Das scheint das BVerfGE 118, 79 (110 f.) zu übersehen.

$40 P$. Graichen/T. Requate, Der steinige Weg von der Theorie in die Praxis des Emissionshandels - Die EURichtlinie zum CO2-Emissionshandel und ihre nationale Umsetzung, Perspektiven der Wirtschaftspolitik 6 (2005), 41 (50). 
um die Entwicklung neuer Klimaschutztechnologien zu beschleunigen. ${ }^{41}$ Man darf nur nicht die Vorstellung haben, dem Klima würde damit schon jetzt etwas Gutes getan.

Das Emissionshandelsrecht ist mithin nicht auf einen funktionierenden Emissionshandel angewiesen, um bestimmte Klimaschutzziele zu erreichen. Aber umgekehrt hängt die Funktionsfähigkeit des Emissionshandels in gewissem Umfang von der planerischen Festlegung des Klimaschutzzieles ab. Denn erst die Begrenzung der Emissionen sorgt für die Knappheit der Emissionsberechtigungen auf dem Markt. Setzt man deshalb, wie in der ersten Emissionshandelsperiode 2005 bis 2007 geschehen, das Kontingent so groß fest, dass es mehr Berechtigungen enthält als tatsächlich Emissionen freigesetzt werden, wird zwar immer noch das Emissionsziel erreicht. Der Emissionsmarkt bricht aber mangels Knappheit zusammen. ${ }^{42}$

\section{Die Zuteilungsregeln: Anreize und Kostenverteilung}

Sowohl das ordnungsrechtliche Fundament, als auch die Modifikationen, die für die Handelbarkeit der Emissionsrechte notwendig sind, werfen zwar eine Vielzahl juristischer Probleme auf, ${ }^{43}$ bergen aber keine grundsätzlichen Rationalitätskonflikte. Man muss sich dazu verhalten, ob die zusätzlichen Freiheitseingriffe grundrechtlich gerechtfertigt werden können, und natürlich stellt sich die Frage des Bestandsschutzes für nach dem BImschG genehmigte Altanlagen. ${ }^{44}$ Die ökonomische Zielsetzung stellt aber kein Hindernis dar, um die modifizierten Betreiberpflichten in die Dogmatik des Umweltrechts zu integrieren. Für die Auslegung und Anwendung dieser Regelungen braucht man auch keinen ökonomischen Sachverstand. Anders liegt es dagegen bei den Regelungen über die Zuteilung von Emissionsberechtigungen, die die Gerichte in erheblichem Maß beschäftigt haben. Die Zuteilungsregeln sind ein echtes Novum des Emissionshandelsrechts. Sie geben aber leider auch ein mahnendes Beispiel, wie rechtliche und ökonomische Rationalität leiden, wenn sich die Jurisprudenz auf einen strikt juristischen Standpunkt zurückzieht und Eigengesetzlichkeiten des ökonomischen Instruments nicht zur Kenntnis nimmt. Denn selbst eine juristische Kernkompetenz wie die kunstgerechte Auslegung gesetzlicher Regelungen kann hier ohne Verständnis der ökonomischen Zusammenhänge nicht voll wahrgenommen werden. ${ }^{45}$

41 Ebd.

42 Deutsche Emissionshandelsstelle im Umweltbundesamt, Emissionshandel, Auswertung der ersten Handelsperiode 2005-2007, 2009, 94 ff. und 123 ff.; Ch. Engel, Rationale Rechtspolitik und ihre Grenzen, JZ 2005, 581 (584).

43 Monographisch behandelt etwa bei M. Diehr, Rechtsschutz im Emissionszertifikate-Handelssystem, 2006, Knüll (Fn. 5); Mehrbrey (Fn. 6); Hohmuth (Fn. 18); T. Zimmer, CO2-Emissionsrechtehandel in der EU, 2004; zu aktuellen Problemen siehe Enders (Fn. 8), 196 ff.; J. Günther/J. Schnutenhaus, Die Rechtsprechung zum Emissionshandelsrecht, NVwZ 2007, $1140 \mathrm{ff}$.

44 Vgl. etwa BVerfGE 118, 79 (94 ff.); BVerfG (Kammer), NVwZ 2007, 942 (945); BVerwGE 124, 47 (58 ff.); M. Burgi, Die Rechtstellung der Unternehmen im Emissionshandelssystem, NJW 2003, 2486 (2490 ff.); Diehr (Fn. 43), 178 ff. und 238 ff.; Enders (Fn. 8), 196 ff.; Knüll (Fn. 5), 282 ff.; Mehrbrey (Fn. 6), 45 ff.; Hohmuth (Fn. 18) 267 ff.; Zimmer (Fn. 43), 207 ff.

45 Insoweit ist es so bezeichnend wie begrüßenswert, dass sich die vorliegenden Dissertationen zum Emissionshandelsrecht zunächst der ökonomischen Zusammenhänge vergewissern; Diehr (Fn. 43), 24 ff.; Knüll (Fn. 5), 39 ff.; Hohmuth (Fn. 18) 15 ff.; Mehrbrey (Fn. 6), 21 ff.; Zimmer (Fn. 43), 67 ff. Diese Einsichten werden, wie sich zeigen wird, von der Rspr. nicht immer berücksichtigt. 


\section{Erstallokation durch Versteigerung oder Zuteilung}

Zunächst: Warum bedarf es überhaupt einer Zuteilung von Emissionsberechtigungen? Weil der Emissionshandel selbst nur für eine Umverteilung der Emissionsberechtigungen sorgt, ökonomisch gesprochen, für deren Endallokation. ${ }^{46}$ Diese Aufgabe erfüllt der Markt allerdings von unsichtbarer Hand, ${ }^{47}$ wenn der Wettbewerb nicht beeinträchtigt ist. ${ }^{48}$ Zuvor aber müssen die Berechtigungen überhaupt in den Markt kommen, und das heißt, der Staat muss deren Erstallokation organisieren. Dafür gibt es im Wesentlichen zwei Wege: kostenlose Zuteilung durch Verwaltungsakt oder entgeltliche Veräußerung durch Verkauf oder Versteigerung. Niemand wird es wundern, dass die ökonomische Literatur fast einhellig für Auktionen plädiert. ${ }^{49}$ Und Auktionen haben in der Tat einige vorteilhafte Eigenschaften: Sie sorgen für ein schnelles Preissignal, ${ }^{50}$ sie beugen Marktmanipulationen vor, ${ }^{51}$ sie verhindern, dass Unternehmen durch die Zuteilungen bereichert werden, ${ }^{52}$ und sie wirken Wettbewerbsverzerrungen innerhalb des Emissionshandelsbereichs entgegen. ${ }^{53}$ Dem stehen allerdings Probleme gegenüber, die für das Recht bedeutsam sind. Eine vollständige Verauktionierung des Emissionskontingents würde jedenfalls einen Teil der Unternehmen mit erheblichen Kosten belasten, ${ }^{54}$ was unter dem Gesichtspunkt des grundrechtlichen Bestandsschutzes für genehmigte Anlagen nicht unbedenklich ist. Außerdem würden Wettbewerbsnachteile entstehen gegenüber Anlagen, die von ihren Mitgliedsstaaten kostenlose Zuteilungen erhalten oder die nicht dem europäischen Emissionshandel unterliegen. ${ }^{55}$ Hier liegt also ein echter, wenngleich lokaler Konflikt zwischen ökonomischer und rechtlicher Rationalität vor, der bislang zugunsten des Rechts entschieden wurde. Denn in der ersten Handelsperiode 2005 bis 2007 wurden sämtliche Berechtigungen durch Verwaltungsakt kostenlos zugeteilt, und in der laufenden Handelsperiode von 2008 bis 2012 immerhin noch über 90 \% (§ 19 ZuG 2012). In welchem Ausmaß allerdings die Rationalität des ökonomischen Steuerungsmechanismus durch eine kostenlose Zuteilung beeinträchtigt wird, hängt entscheidend von der gewählten $\mathrm{Zu}$ -

$46 \quad$ Endres (Fn. 6), $130 \mathrm{f}$.

47 Montgomery (Fn. 6); Weimann (Fn. 6), 228;

48 Cansier (Fn. 6), 195 f. Beeinträchtigungen des Emissionsmarktes können insbesondere durch hohe Transaktionskosten oder eine geringe Zahl der Marktteilnehmer entstehen.

49 C. Hepburn, Carbon Trading, Annual Review of Environment and Resources 32 (2007), 375 (389): ,,a very strong conclusion from the economic literature is that a large proportion of the cap should be auctioned."; ebenso z. B. Ch. Böhringer/A. Lange, Mission Impossible? On the Harmonization of National Allocation Plans under the EU Emissions Trading Directive, Journal of Regulatory Economics 27 (2005), $81 \mathrm{ff}$.; Ch. Böhringer/A. Lange/U. Moslener, Der EU-Emissionshandel im Zielkonflikt zwischen Effizienz, Kompensation und Wettbewerbsneutralität, Perspektiven der Wirtschaftspolitik 6 (2005), 309 (321); Cansier (Fn. 6), 188 ff; Graichen/Requate (Fn. 40), 46; C. Hepburn/M. Grubb/K. Neuhoff/F. Mathes/M. Tse, Auctioning of EU ETS Phase II Allowances: How and Why, Climate Policy 6 (2006), 137 ff.; T. Voigt/B. Hansjürgens, Der Emissionshandel in Deutschland - Eine Bewertung aus ökonomischer Sicht, in: Immissionsschutz durch Emissionshandel (Fn. 35), 37 (45); Weimann (Fn. 6), 232.

$50 \quad$ Graichen/Requate (Fn. 40), 46.

51 Weimann (Fn. 6), 232.

52 C. Hepburn et al. (Fn. 49), 140.

53 Böhringer/Lange/Moslener (Fn. 49), 321. Für eine Diskussion weiterer Vor- und Nachteile vgl. C. Hepburn et al. (Fn. 49), $138 \mathrm{ff}$.

54 D. Burtraw/K. Palmer, Compensation Rules for Climate Policy in the Electricity Sector, Journal of Policy Analysis and Management 27 (2008), 819 (820). Zu den Kostenfolgen der Zuteilung siehe noch ausführlich unten, S. 14.

55 C. Hepburn et al. (Fn. 49), 141. 
teilungsmethode und ihrer rechtlichen Ausgestaltung ab, ${ }^{56}$ und hier trifft man auf unnötige Missverständnisse und Fehlgestaltungen.

\section{Zuteilungen als Modernisierungsanreize?}

Bei der juristischen Interpretation der Zuteilungsregeln trifft man an prominenter Stelle auf die Vorstellung, mit der Zuteilung oder Vorenthaltung von Berechtigungen würden Anreize zur Modernisierung gesetzt. Das Bundesverfassungsgericht greift zentral auf diesen Gedanken zurück, um die ungleiche Behandlung verschiedener Gruppen von Bestandsanlagen zu rechtfertigen, nämlich einerseits solcher, die bereits vor Einführung des Emissionshandels freiwillig modernisiert worden waren (sog. early actions), und andererseits solcher, die erst unter dem Emissionshandelssystems modernisiert wurden oder werden (sog. late actions). Nach dem ZuG 2007 erhielten alle Bestandsanlagen im Grundsatz Zuteilungen in Höhe ihrer früheren Emissionen in einer bestimmten Basisperiode vor Einführung des Emissionshandels, die um einen Faktor von insg. 7,4 \% anteilig gekürzt wurden ( $\S 7$ I, 5, 4 IV ZuG 2007). Ohne weitere Regelungen wären durch diese Zuteilungsmethode frühe Emissionsminderungen gegenüber späten jedenfalls insoweit benachteiligt worden, als Betreiber frühzeitig modernisierter Anlagen weniger Möglichkeiten haben, durch weitere Modernisierungsmaßnehmen Emissionsberechtigungen freizusetzen und diese am Emissionsmarkt gewinnbringend zu verkaufen. Bei ihnen wurde nämlich das Potential für kostengünstige Emissionsreduzierungen schon vor Einführung des Emissionshandels teilweise ausgeschöpft. ${ }^{57}$ Aus diesem Grund stellte $\S 12$ ZuG 2012 frühzeitige Modernisierungsmaßnahmen für einen bestimmten Zeitraum von den anteiligen Kürzungen frei. Das Bundesverfassungsgericht hatte nun unter anderem die Frage $\mathrm{zu}$ beantworten, ob die verbleibende Ungleichbehandlung von Art. 3 I GG Bestand hat.

Das Gericht bejahte dies unter anderem mit dem Argument, der Gesetzgeber dürfe „für künftige Modernisierungsmaßnahmen besondere Anreize vorsehen, zumal dann, wenn die erreichten Reduktionen von beträchtlichem Ausmaß sind. ${ }^{* 58}$ Es scheint insoweit anzunehmen, die Zuteilung von Berechtigungen wirke wie eine gezielte Subvention von Reduktionsmaßnahmen. Unter Berufung auf seine Rechtsprechung zur gleichheitsrechtlichen Beurteilung von Steuern mit Lenkungszielen ${ }^{59}$ führt es aus: „Vergleichbar der Situation im Abgabenrecht ist es dem Gesetzgeber auch bei der Zuteilung von Emissionsberechtigungen gestattet, ein - dem gesetzgeberischen

56 Ch. Böhringer/A. Lange, On the Design of Optimal Grandfathering Schemes for Emission Allowances, European Economic Review 49 (2005), 2041 ff.; K. E. Rosendahl, Incentives and Prices in an Emissions Trading Scheme with Updating, Journal of Environmental Economics and Management 56 (2008), 69 ff.; Th. Sterner/A. Muller, Output and Abatement Effects of Allocation Readjustment in Permit Trade, Climatic Change 86 (2008), $33 \mathrm{ff}$.

57 Diehr (Fn. 43), 251. Dagegen liegt eine Benachteiligung nicht schon darin, dass frühzeitig modernisierte Anlagen bezogen auf eine bestimmte Produktion eine geringere Anzahl von Berechtigungen erhalten, weil auch ihr Bedarf an Berechtigungen geringer ist.

58 BVerfGE 118, 79 (107).

59 BVerfGE 110, 274 (292 ff.). 
Lenkungszweck entsprechendes - erwünschtes Verhalten der Normunterworfenen zu fördern. “60 Der Gesetzgeber dürfe nicht nur durch Ge- und Verbote, sondern auch ,durch die Belastung eines unerwünschten oder durch Privilegierung eines erwünschten Verhaltens“ steuernd auf Wirtschaft und Gesellschaft Einfluss nehmen. ${ }^{61}$ Auch in der kostenlosen Zuteilung von Berechtigungen erblickt das Gericht eine solche verhaltenslenkende Privilegierung von Modernisierungsmaßnahmen. Die durch eine Emissionsreduktion freigesetzten Zertifikate könnten am Markt veräußert werden und so indirekt zur Finanzierung der Modernisierungsmaßnahmen beitragen. ${ }^{62}$ Auch im Zusammenhang der Frage, ob die Zuteilungsregeln des ZuG 2007 der verfassungsrechtlichen Pflicht zum Schutz der natürlichen Lebensgrundlagen aus Art. 20a GG genügen, meint das Gericht, um eine Reduktion von Treibhausgasen zu erreichen, sei es notwendig, durch Zuteilungsregeln ,weitere Anreize für Modernisierungen zu schaffen““63

\section{Anreizneutralität der anfänglichen Zuteilung von Berechtigungen}

Nun ist der Gedanke auf den ersten Blick in der Tat plausibel und naheliegend, eine kostenlose Zuteilung von Berechtigungen setze einen Anreiz zur Modernisierung, weil die Betreiber freiwerdende Emissionsberechtigungen dann gewinnbringend veräußern können. Betrachtet man die Anreizwirkungen des Emissionshandels genauer, stellt man aber fest, dass der Modernisierungsanreiz überhaupt nicht auf der Zuteilung beruht. Tatsächlich ist es für die Modernisierungsentscheidung völlig irrelevant, ob ein Unternehmen über Berechtigungen verfügt oder nicht. Dass man kostenlos zugeteilte Emissionsberechtigungen nach einer Modernisierung verkaufen kann, ist nämlich nur einer der beiden Gründe, über die das Emissionshandelssystem Unternehmen einen Anreiz zur Modernisierung gibt. Für Unternehmen, die keine kostenlosen Emissionsberechtigungen erhalten haben, besteht ein anderer, gleichwertiger Modernisierungsgrund, nämlich die Kosten für den Zukauf von Emissionsberechtigungen zu sparen. Die Voraussetzungen, unter denen sich eine Modernisierung lohnt, sind in beiden Fällen nun exakt die gleichen, nämlich dass die Modernisierung geringere Kosten verursacht, als der Marktwert der eingesparten Emissionsberechtigungen beträgt. ${ }^{64}$ Das heißt, für die Modernisierungsentscheidung kommt es auf bereits erhaltene oder nicht erhaltene Zuteilungen schlicht nicht an. Selbst wenn alle Berechtigungen zu Beginn der Handelsperiode dem Heizkraftwerk des Bonner Universitätsklinikums zugeteilt worden wären, wären die Modernisierungsanreize im Prinzip die gleichen geblieben. ${ }^{65}$ Wie Lenkungssubventionen kann man die Zuteilungen also in der Regel nicht verstehen (zu den Ausnahmen sogleich), so dass eine Bevorzugung von späten gegenüber frühen Emissionsminderun-

60 BVerfGE 118, 79 (105). Es ist allerdings nicht klar, ob sich diese Aussage nur auf die Prüfung der Ersatzanlagenregelung es $\S 10 \mathrm{ZuG} 2007$ bezieht, in deren Zusammenhang sie getroffen wurde, oder allgemeiner gemeint ist. Bezüglich der Ersatzanlagenregelung beruht sie zwar immer noch auf einem Missverständnis, ist aber nicht grundlegend falsch, wie noch dargelegt wird.

61 Ebd.

62 BVerfGE 118, $79(106)$.

63 BVerfGE 118, 79 (110 f.).

64 C. Stewing/G. Walther, Maßnahmen zur Emissionsminderung, in: M. Elspas et al. (Hg.), Emissionshandel, 2006, Kap. 24, Rn. 45.

65 Vgl. Sterner (Fn. 6), 90. 
gen, wenn sie denn vorlag, vor Art. 3 I GG jedenfalls nicht durch den Lenkungszweck gerechtfertigt werden kann. Was dabei übersehen wurde, ist ein grundsätzlicher Unterschied in der Funktionsweise des Emissionshandels im Vergleich zu Lenkungssubventionen oder -steuern. Letztere beeinflussen Verhalten dadurch, dass der Staat gewünschtes Verhalten gezielt subventioniert bzw. unerwünschtes Verhalten gezielt verteuert. Die Anreize des Emissionshandels für kosteneffiziente Modernisierungen beruhen dagegen darauf, dass der Staat „Eigentumsrechte“ schafft, die unabhängig von dem Emissionsverhalten der Unternehmen Bestand haben. ${ }^{66}$ Der Emissionshandel schafft die gewünschten Anreize also gerade dadurch, dass an die Modernisierungsentscheidung keine Konsequenzen geknüpft sind, sondern die Zuteilung unverändert bleibt. ${ }^{67}$ Unter dieser Voraussetzung richtet sich die Modernisierungsentscheidung, wie gesagt, nur danach, ob die Grenzvermeidungskosten des Betreibers oder der Marktpreis für Berechtigungen höher sind. Da die Höhe der anfänglichen Zuteilung darauf keinen Einfluss hat, ist die Zuteilungsmethode im Grundsatz anreizneutral. ${ }^{68}$

\section{Besondere Modernisierungsanreize}

Davon gibt es allerdings eine gewichtige Ausnahme, die auch für die juristische Interpretation der Zuteilungsregeln bedeutsam ist. Sie betrifft eben die Fälle, in denen die Zuteilungsregeln entgegen der Grundkonzeption des Emissionshandels so ausgestaltet werden, dass sie die Eigenschaften von Lenkungsanreizen annehmen. Dazu kommt es, wenn das Verhalten eines Betreibers nach der Zuteilungsregel Einfluss auf die Höhe der Zuteilung hat, sei es, dass es zu einer nachträglichen Änderung der Zuteilungsentscheidung führt, sei es, dass es für die Höhe der Zuteilung in der nächsten Handelsperiode relevant ist. ${ }^{69}$ Solche Zuteilungsregeln können nun in der Tat „besondere“, von den systemimmanenten Anreizen des Emissionshandels abweichende Anreize für oder gegen eine Modernisierung setzen. Solche besonderen Modernisierungsanreize können unbeabsichtigt in scheinbar unverfänglichen Regelungen enhalten sein (wie in dem nachfolgenden Beispiel der Stillegungsregelung), oder bewusst geschaffen werden, weil der Gesetzgeber meint, auf diese Weise den Klimaschutz fördern zu können. Tatsächlich sind besondere Modernisierungsanreize wegen des gleichbleibenden Kontingents für den Klimaschutz nutzlos, aber auch nicht schädlich. Sie beeinträchtigen aber die Kosteneffizienz des Emissionshandels und können im ungünstigen Fall sogar die beabsichtigten Modernisierungsanreize des Emissionshandels außer Kraft setzen.

Ein anschauliches Beispiel dafür ist die Stilllegungsregelung (§§ $9 \mathrm{ZuG} 2007,10 \mathrm{ZuG} 2012$ ). Sie betrifft die Frage, was mit zugeteilten Berechtigungen passiert, wenn der Betrieb einer Anlage vor dem Ende der Handelsperiode eingestellt wird. Aus juristischer Sicht erscheint es nahelie-

66 Endres (Fn. 6), $101 \mathrm{ff}$.

67 M. Ahman/D. Butraw/J. Kruger/L. Zetterberg, A Ten-Year Rule to Guide the Allocation of EU Emission Allowances, Energy Policy 35 (2007), 1718 (1721); Böhringer/Lange (Fn. 56), 2042; Rosendahl (Fn. 56), $69 \mathrm{f}$.

68 Weimann (Fn. 6), $229 \mathrm{ff}$.

69 Ahman et al. (Fn. 67) 1721; Böhringer/Lange (Fn. 56), 2042; Rosendahl (Fn. 56), 69 f. 
gend, dass in diesem Fall die Zuteilungsentscheidung widerrufen und die Berechtigungen zurückzugeben sind, wie es die Zuteilungsgesetze auch vorsehen. Andernfalls wäre der Betreiber durch die nicht mehr benötigten Berechtigungen ohne Grund bereichert. Eine solche Regelung beeinträchtigt auch nicht notwendig die Kosteneffizienz des Emissionshandels, wenn die Stilllegung aus Anlass einer Produktionsreduktion erfolgt. ${ }^{70}$ Ganz anders sieht es aber aus, wenn eine klimaschädliche alte Anlage durch eine neue und effizientere Anlage ersetzt werden soll, also in einer der Konstellationen, für die der Emissionshandel wesentlich geschaffen wurde. Indem das Gesetz dem Betreiber in dieser Situation die Berechtigungen für die alte Anlage nimmt, zerstört es den Anreiz, den der Emissionshandel eigentlich schaffen soll, weil der Betreiber die Modernisierung dann nicht mehr durch den Verkauf freigewordener Berechtigungen finanzieren kann. ${ }^{71}$

Ohne diese Einsicht im Hinterkopf, erschließt sich auch der Sinn einer weiteren Regelung nicht, nämlich der sog. Ersatzanlagenregelung ( $\$ 10 \mathrm{ZuG}$ 2007). Diese in der ersten Handelsperiode geltende Regelung bestimmte, dass ein Betreiber für vier Jahre die Zuteilungen für eine stillgelegte Anlage weiter erhält, sofern er diese durch eine moderne neue Anlage ersetzt hat. Rechtsprechung und Literatur haben diese Regelung vor allem als einen besonderen, zusätzlichen Modernisierungsanreiz verstanden, der Ersatzanlagen wegen besonderer Beiträge zum Klimaschutz gegenüber anderen Modernisierungsmaßnahmen zusätzlich begünstigt. ${ }^{72}$ Die Gesetzesbegründung meint, mit dieser Regelung einen Anreiz ,zur beschleunigten Inbetriebnahme zukunftsorientierter, hoch effizienter Anlagen“ zu schaffen. ${ }^{73}$ Daran anknüpfend rechtfertigt das Bundesverfassungsgericht die Regelung gegenüber einem möglichen Gleichheitsverstoß mit dem Argument, die Ersatzanlagenregelung habe in besonderem Maße eine Minderung der Treibhausgasemissionen im Blick. ${ }^{74}$ Nach dem Gesagten ist klar, dass diese Auffassung auf einem grundlegenden Missverständnis über die Anreizwirkungen von Zuteilungsregeln beruht. Tatsächlich enthält die Ersatzanlagenregelung keine zusätzlichen Modernisierungsanreize. Sie setzt nur diejenigen allgemeinen, systemimmanenten Anreize des Emissionshandels wieder in Kraft, und dies auch nur zum Teil, die zuvor durch die Stilllegungsregelung eliminiert worden waren. Sie sorgt dafür, dass der Emissionshandelsmechanismus insoweit wenigstens zum Teil überhaupt funktioniert.

Nun hatte der Umweltausschuss in den Beratungen zum ZuG 2012 mittlerweile erkannt, dass die Stilllegungsregelung ein Problem darstellt, weil sie einen „Anreiz zum Weiterbetrieb veralteter Kraftwerke“ setzt. ${ }^{75}$ Allerdings führt der Umweltausschuss dieses Problem fälschlicherweise nur

70 Vorausgesetzt, der Betreiber kann nicht zwischen mehrere Anlagen mit unterschiedlichem Emissionsstandards auswählen. Ist das der Fall, führt die Stilllegungsregelung dazu, dass die unterschiedlichen Emissionskosten der Anlagen (in Höhe der kostenlosen Zuteilungen) bei der Auswahl zwischen den Anlagen nicht berücksichtigt werden. Das kann zur Folge haben, dass eine ineffizientere Anlage mit höheren Emissionskosten weiter betrieben wird, obwohl sie, wenn die Emissionskosten berücksichtigt würden, auch für den Betreiber höhere Kosten verursacht, wie Ahman et al. (Fn. 67), 1721 ff., gezeigt haben.

71 Ebd.

$72 \quad$ W. Frenz, Natur und Recht 29 (2007), S. 513 (518).

73 BT-Drucks. 15/2966, S. 17, ähnlich S. 21. Andere Gründe werden dort nicht genannt.

74 BVerfGE 118, 79 (103 ff.).

75 Beschlussempfehlung des Ausschusses für Umwelt, Naturschutz und Reaktorsicherheit, BT-Drucks. 16/5769, S. 17. 
auf die kostenlose Zuteilung zurück und empfiehlt deshalb die teilweise entgeltliche Veräußerung von Berechtigungen im Kraftwerksbereich. Dabei übersieht er leider, dass gerade bei den Kraftwerken das Problem der Stilllegungsregelung bereits durch die Einführung einer Zuteilung nach normativen Emissionswerten weitgehend behoben worden war ( $\$ 7 \mathrm{ZuG} 2012$ ). ${ }^{76}$ Ausgerechnet dort aber, wo das Problem tatsächlich noch fortbestand, nämlich bei den Industrieanlagen, wurde die Ersatzanlagenregelung, die das Problem bislang abgemildert hatte, aus Vereinfachungsgründen gestrichen. Hier werden also wichtige Anreizeffekte übersehen und dadurch die beabsichtigten Wirkungen des Emissionshandels konterkariert.

\section{Zuteilungsregeln als Kostenverteilungsregeln}

Auch wenn die Zuteilungsregeln anreizneutral ausgestaltet werden, wie es nach der ökonomischen Rationalität wünschenswert ist, sie sind alles andere als irrelavant, insbesondere nicht für das Recht. Sie bestimmen nämlich darüber, inwieweit die Betreiber mit den Emissionskosten belastet werden und wie diese zwischen den Betreibern verteilt werden. Isoliert betrachtet beinhaltet jede kostenlose Zuteilung zunächst eimal ein Kapitalgeschenk. ${ }^{77}$ So wäre die Universität Bonn bei einer Zuteilung sämtlicher Berechtigungen an ihr Heizkraftwerk um gut 12 Milliarden Euro pro Jahr reicher gewesen. ${ }^{78}$ Allerdings verursachen $\mathrm{CO}_{2}$-Emissionen seit der Einführung des Emissionshandels auch Kosten, ${ }^{79}$ entweder für den Zukauf von Berechtigungen, oder Opportunitätskosten, weil verbrauchte eigene Berechtigungen nicht mehr auf dem Markt verkauft werden können. ${ }^{80}$ Diese Emissionskosten werden nun aber durch die kostenlosen Zuteilungen kompensiert, so dass die Unternehmen in diesem Umfang durch den Emissionshandel nicht belastet werden. Soweit die kostenlosen Zuteilungen dagegen hinter den tatsächlichen Emissionen einer Anlage zurückbleiben, fallen bei den Unternehmen Emissionskosten tatsächlich an. ${ }^{81}$ Die we-

76 Nach dieser Regelung richtet sich auch die Zuteilung an Bestandsanlagen nicht mehr nach den tatsächlichen Emissionen in einer historischen Basisperiode, sondern danach, wie viel Emissionsberechtigungen für eine entsprechende Produktion bei Zugrundelegung normativer (am Stand der Technik orientierter) Emissionswerte benötigt werden. Damit wird insoweit die gleiche Zuteilungsmethode eingesetzt, wie sie gemäß $\S 9$ ZuG 2012 auch für Neuanlagen gilt, und diese richtet sich nicht nach der Emissions-, sondern nach der Produktionsmenge. Sieht man von Unterschieden bei der Bestimmung der relevanten Produktionsmenge ab, bleibt bei dieser Rechtslage die Zuteilung von der Ersetzung einer alten durch eine neue Anlage unberührt. Allerdings kann ein Anreiz zur Überproduktion entstehen, wenn die Zuteilung höher ist als die Emissionen, die bei kosteneffizienter Vermeidungstechnologie anfallen; vgl. Böhringer/Lange (Fn. 56); Rosendahl (Fn. 56).

77 Ahman et al. (Fn. 67), 1720.

78 Bei einem Berechtigungspreis von $15 €$ und einer Menge von 844 Mio. Berechtigungen.

79 Soweit der Marktpreis für Berechtigungen auch die sozialen Kosten der Emissionen abbildet (was gegenwärtig aber wohl nur zum Teil der Fall ist; s.o., Fn. 33), werden insoweit auch die externen Kosten internalisiert; vgl. Menges (Fn. 26), 285 f. u. $288 \mathrm{f}$.

80 Ahman et al. (Fn. 67), 1720; H. Friederiszick/L.-H. Röller, Überwälzungen der Opportunitätskosten von CO2-Zertifikaten als Ausbeutungsmissbrauch - eine ökonomische Analyse, Wirtuschaft und Wettbewerb 2008, 929 (931 f.); Woerdman/A. Arcuri/S. Clò, Emissions Trading and the Polluter-Pays Principle, Review of Law and Economics 4 (2008), 565 (576 ff.).

81 Sie müssen dann entweder Aufwendungen zur Emissionsvermeidung treffen oder Emissionsberechtigungen zukaufen. 
sentliche Wirkung der anfänglichen Zuteilung besteht also darin, den individuellen Betreiber in Höhe seiner Unterdeckung mit Emissionskosten zu belasten. ${ }^{82}$

Interpretiert man Zuteilungsregeln dagegen als Anreizregeln, obwohl sie tatsächlich nur die Kosten hin und her schieben, übersieht man nicht nur die ökonomischen Sachzusammenhänge, sondern man verfehlt auch den juristischen Sinn der Regelung. Denn wo sich das Recht ausdrücklich die Umsetzung eines ökonomischen Steuerungsmodells zur Aufgabe gemacht hat, kann es die Logik dieses Modells nicht willkürlich ignorieren. Das schließt selbstverständlich nicht aus, dass sich der Gesetzgeber zugunsten anderer Gemeinwohlbelange über Effizienzerwägungen hinwegsetzt und insoweit die ökonomische Rationalität zurücktreten lässt. ${ }^{83}$ Die Jurisprudenz verkürzt aber ihre eigene Rationalität ohne Not, wenn sie Regelungen ökonomische Funktionen zuschreibt, die diese gar nicht haben, und deshalb Spielräume unbeachtet lässt, innerhalb derer sich genuin rechtlichen Belange auch ohne Konflikte mit der ökonomischen Rationalität zur Geltung bringen ließen. Der Emissionshandel ist insoweit ein Steuerungsmodell, das auch der rechtlichen Rationalität entgegen kommt. Es erlaubt nämlich eine partielle Separierung von Anreizwirkung und Lastenverteilung, die es ermöglicht, beide Rationalitäten unabhängig voneinander zu verfolgen, solange bestimmte Fehler vermieden werden.

\section{Resümee}

Die Zuteilungsregeln böten noch reichlich Anschauungsmaterial, um diese Befunde zu vertiefen. An dieser Stelle soll aber ein vorläufiges Resümee genügen. Die Prozeduralisierung der ordnungsrechtlichen Betreiberpflichten ist ein Beispiel dafür, wie ökonomische Steuerungsmechanismen in das öffentliche Recht und seine Dogmatik integriert werden können, ohne deren Eigenrationalität zu korrumpieren. Die Zuteilungsregeln machen aber deutlich, dass auch Anstrengungen geboten sind, damit eine Vermengung der Rationalitäten gelingt. So geht rechtliche Rationalität verloren, wenn in Unkenntnis der ökonomischen Zusammenhänge Normen fehlgedeutet und rechtliche Gestaltungsräume übersehen werden. Auch sollte das Recht Folgewirkungen bedenken, die aufgrund der ökonomischen Eigengesetzlichkeiten vorhersehbar und für die gesetzgeberischen Ziele relevant sind. Nur dann lassen sich unnötige Beeinträchtigungen von Rechtswerten oder Effizienz vermeiden und nur dann können unvermeidbare Restkonflikte in einer für beide Rationalitäten schonenden Weise gelöst werden.

82 Sie berücksichtigen ist aber auch, dass manche Unternehmen Emissionskosten (je nach Industrie in unterschiedlichem Umfang) zum Teil auf die Produktpreise überwälzen können; Ahman et. al (Fn. 67); Friederiszick/Röller (Fn. 80), 932 ff. Insoweit werden Emissionskosten dann nicht von den Anlagenbetreibern, sondern von deren Abnehmern bezahlt. Weil eine solche Überwälzung auch bei Opportunitätskosten möglich ist, können Betreiber durch kostenlose Zuteilungen sogar erheblich bereichert werden, wie dies im Bereich der Stromerzeugen der Fall war; vgl. W. Frenz, Kostenlose Emissionszertifikate und unangemessene Preise, Wirtschaft und Wettbewerb 2006, 737 ff.; Friederiszick/Röller (Fn. 80). Eine zusätzliche Kompensation für Emissionskosten können solche Unternehmen erreichen, die ihre Emissionen über die Unterdeckung hinaus reduzieren und die frei werdenden Berechtigungen mit Gewinn veräußern können (ohne dies würde kein Emissionshandel stattfinden). 


\section{Preprints 2009}

Stephen N. Broadberry / Carsten Burhop: Real Wages and Labour Productivity in Britain and Germany, 1871-1938: A Unified Approach to the International Comparison of Living Standards. 2009/18.

Andreas Glöckner / Sara D. Hodges: Parallel Constraint Satisfaction in Memory-Based Decisions. 2009/17.

Niels Petersen: Review Essay - How Rational is International Law? 2009/16.

Felix Bierbrauer: On the legitimacy of coercion for the financing of public goods. 2009/15.

Feri / Irlenbusch / Sutter: Efficiency Gains from Team-Based Coordination - Large-Scale Experimental Evidence.2009/14.

Jos Jansen: On Competition and the Strategic Management of Intellectual Property in Oligopoly. 2009/13.

Martin Hellwig: Utilitarian Mechanism Design for an Excludable Public Good. 2009/12.

Philipp Weinschenk: Persistence of Monopoly and Research Specialization. 2009/11.

Nina Horstmann/Andrea Ahlgrimm/Andreas Glöckner: How Distinct are Intuition and Deliberation? An Eye-Tracking Analysis of Instruction-induced Decision Modes. 2009/10.

Thorsten Lübbers: Is Cartelisation Profitable? A Case Study of the Rhenish Westphalian Coal Syndicate, 1893-1913. $2009 / 9$.

Andreas Glöckner/Bernd Irlenbusch/Sebastian Kube/Andreas Nicklisch/Hans-Theo Normann: Leading with(out) Sacrifice? A Public-Goods Experiment with a Super-Additive Player. 2009/8.

Carl Christian von Weizsäcker: Asymmetrie der Märkte und Wettbewerbsfreiheit. 2009/7.

Jos Jansen: Strategic Information Disclosure and Competition for an Imperfectly Protected Innovation. 2009/6.

forthcoming in: Journal of Industrial Economics

Niels Petersen: Abkehr von der internationalen Gemeinschaft? - Die aktuelle Rechtsprechung des US Supreme Court zur innerstaatlichen Wirkung von völkerrechtlichen Verträgen. 2009/5.

Johannes Rincke / Christian Traxler: Deterrence Through Word of Mouth. 2009/4.

Christian Traxler / Joachim Winter: Survey Evidence on Conditional Norm Enforcement. 2009/3.

Britta Herbig / Andreas Glöckner: Experts and Decision Making: First Steps Towards a Unifying Theory of Decision Making in Novices, Intermediates and Experts. 2009/2.

Martin Beckenkamp: Environmental dilemmas revisited: structural consequences from the angle of institutional ergonomics. 2009/1.

\section{Preprints 2008}

Carsten Burhop: The Underpricing of Initial Public Offerings in Imperial Germany, 1870-1896. 2008/46.

Martin Hellwig: A Note on Deaton's Theorem on the Undesirability of Nonuniform Excise Taxation. 2008/45.

Martin Hellwig: Zur Problematik staatlicher Beschränkungen der Beteiligung und der Einflussnahme von Investoren bei großen Unternehmen. 2008/44.

published in: Zeitschrift für das gesamte Handelsrecht und Wirtschaftsrecht 172 (2008), 768 - 787

Martin Hellwig: Systemic Risk in the Financial Sector: An Analysis of the Subprime-Mortgage Financial Crisis. 2008/43.

Andreas Glöckner / Ann-Katrin Herbold: Information Processing in Decisions under Risk: Evidence for Compensatory Strategies based on Automatic Processes. 2008/42.

Jörn Lüdemann / Stefan Magen: Effizienz statt Gerechtigkeit? 2008/41.

Christoph Engel: Die Bedeutung der Verhaltensökonomie für das Kartellrecht. 2008/40.

Felix Bierbrauer: A unified approach to the revelation of public goods preferences and to optimal income taxation. 2008/39. 
Carsten Burhop / Thorsten Lübbers: Incentives and Innovation? R\&D Management in Germany's High-Tech Industries During the Second Industrial Revolution. 2008/38.

Andreas Nicklisch / Tobias Salz: Reciprocity and status in a virtual field experiment. 2008/37.

Andreas Glöckner / Christoph Engel: Can We Trust Intuitive Jurors? An Experimental Analysis. 2008/36.

Jörn Lüdemann: Wettbewerb und Regulierung in der Telekommunikation. Das Telekommunikationsrecht vor den Herausforderungen dynamischer Märkte. 2008/35.

published in: Jörn Lüdemann (Hrsg.), Telekommunikation, Energie, Eisenbahn - Welche Regulierung brauchen die Netzwirtschaften? Tübingen (Mohr Siebeck) 2008, S. 69-99

Martin Beckenkamp: Playing strategically against nature? - Decisions viewed from a game-theoretic frame. 2008/34.

Christoph Engel: Preponderance of the Evidence versus Intime Conviction. A Behavioural Perspective on a Conflict between American and Continental European Law? 2008/33.

Kristoffel Grechenig: Schadenersatz bei Verletzung von $§ 14 \mathrm{WpHG}$ ? Insiderhandel mit positiver und negativer Information. 2008/32.

Felix Bierbrauer: Optimal Income Taxation, Public Goods Provision and Robust Mechanism Design. 2008/31.

Hans-Theo Normann: Vertical Integration, Raising Rivals' Costs and Upstream Collusion. 2008/30.

forthcoming in: European Economic Review

Martin Hellwig: Competition Policy and Sector-Specific Regulation for Network Industries. 2008/29.

forthcoming in: X. Vives (ed.), "Competition Policy in the EU: Fifty Years on from the Treaty of Rome" Oxford University Press.

Niels Petersen: Rational Choice or Deliberation? - Customary International Law between Coordination and Constitutionalization. 2008/28.

forthcoming in: Journal of Institutional and Theoretical Economics 165 (2009)

Stephen Broadberry / Carsten Burhop: Resolving the Anglo-German Industrial Productivity Puzzle, 1895-1935:

A Response to Professor Ritschl. 2008/27.

forthcoming in: Journal of Economic History

Niels Petersen: Demokratie und Grundgesetz - Veränderungen des Demokratieprinzips in Art. 20 Abs. 2 GG angesichts der Herausforderungen moderner Staatlichkeit. 2008/26.

forthcoming: Jahrbuch des öffentlichen Rechts der Gegenwart 57 (2009)

Andreas Nicklisch: Semi-collusive advertising and pricing in experimental duopolies. 2008/25.

Andreas Nicklisch: Inequity Aversion, Reciprocity, and Appropriateness in the Ultimatum-Revenge Game. 2008/24.

Christoph Engel: The Behaviour of Corporate Actors. A Survey of the Empirical Literature. 2008/23.

Emanuel Vahid Towfigh: Komplexität und Normenklarheit - oder: Gesetze sind für Juristen gemacht. 2008/22.

Christian Traxler / Andreas Reutter: Apportionment, Fiscal Equalization and Decentralized Tax Enforcement. 2008/21.

Christoph Engel, REITs ante portas. Die Anpassung des deutschen Rechts an institutionelle Investoren in den Grundstücks- und Mietmärkten. 2008/20.

forthcoming in: Juristenzeitung 